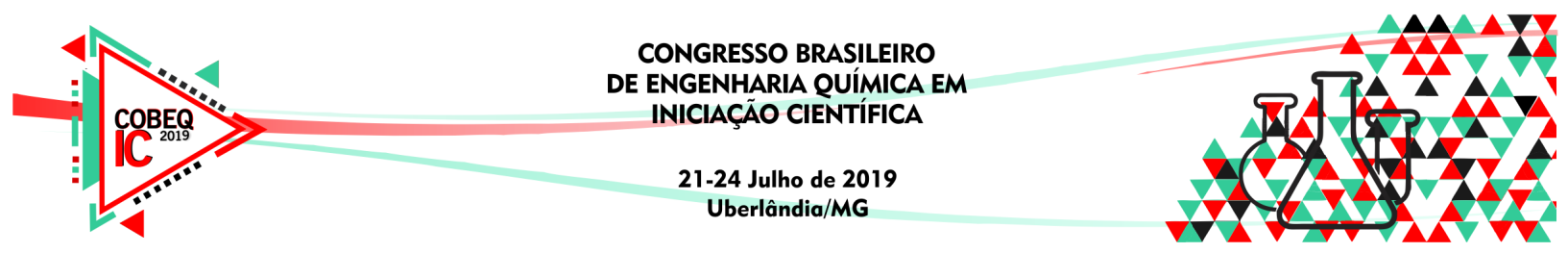

\title{
ESTUDO DAS VARIÁVEIS QUE INFLUENCIAM NA REAÇÃO DE TRANSTERIFICAÇÃO IN SITU DA DESMODESMUS SP.
}

\author{
V. de F. VIEIRA ${ }^{1}$, L. F. RIOS PINTO ${ }^{1, *}$, G.F. FERREIRA ${ }^{1}$ e L.V. FREGOLENTE ${ }^{1}$ \\ ${ }^{1}$ Universidade Estadual de Campinas, Faculdade de Engenharia Química \\ *E-mail para contato: luisa.rpinto@yahoo.com
}

\begin{abstract}
RESUMO - Nos últimos anos, o cultivo de microalgas para produção de óleo tem sido extensivamente estudado. A capacidade de algumas espécies em acumular lipídeos com perfis de ácidos graxos de cadeias contendo de 16 a 18 carbonos as torna potencial matéria prima para a produção de biodiesel. Entretanto, a etapa de extração de lipídeos para produção convencional de óleo de microalgas envolve elevados investimentos e custo de operação. Isto porque há necessidade de rompimento celular, uma vez que as microalgas têm uma parede celular rígida, impedindo o acesso dos solventes aos lipídios acumulados dentro das células. Desta forma, a transesterificação in situ apresenta-se como alternativa interessante, utilizando o álcool tanto como reagente quanto solvente, eliminando a etapa de extração. Neste trabalho, foi proposto o estudo da reação de transesterificação in situ da biomassa da cepa Desmodesmus sp. para a produção de biodiesel. Foram avaliados os efeitos das variáveis temperatura, tempo de reação, volume de álcool e porcentagem de catalisador sobre o rendimento de ésteres metílicos de ácidos graxos. Os resultados mostraram que o parâmetro temperatura de reação é o mais significativo na obtenção de ésteres.
\end{abstract}

\section{INTRODUÇÃO}

A redução de emissões de poluentes atmosféricos tem ocupado uma posição de destaque em conferências internacionais e políticas governamentais nas últimas décadas ( $\mathrm{Su}$ et al., 2017), pois o efeito de acúmulo de gases de efeito estufa e outros poluentes na atmosfera não se limita apenas às mudanças climáticas e outros impactos ambientais, mas também desencadeia uma série de outros graves problemas de ordem econômica e social. Diante da relevância do tema, o desenvolvimento de novos combustíveis de fontes renováveis para substituição de combustíveis fósseis representa um item de alta prioridade na agenda de institutos de pesquisa, empresas de tecnologia e universidades do mundo inteiro.

Nesse contexto, as microalgas despontam como potencial matéria-prima para a produção de biodiesel devido às suas características biológicas, como alta eficiência fotossintética, boa produtividade de biomassa e alto teor de óleo (Su et al., 2017). Além disso, apresentam vantagens econômicas, como possibilidade de cultivo em água salgada ou residual e potencial extração de produtos de alto valor agregado direcionados a outras indústrias, como a farmacêutica, a cosmética e a de alimentos (Oilgae, 2014).

\subsection{Reação de transesterificação}




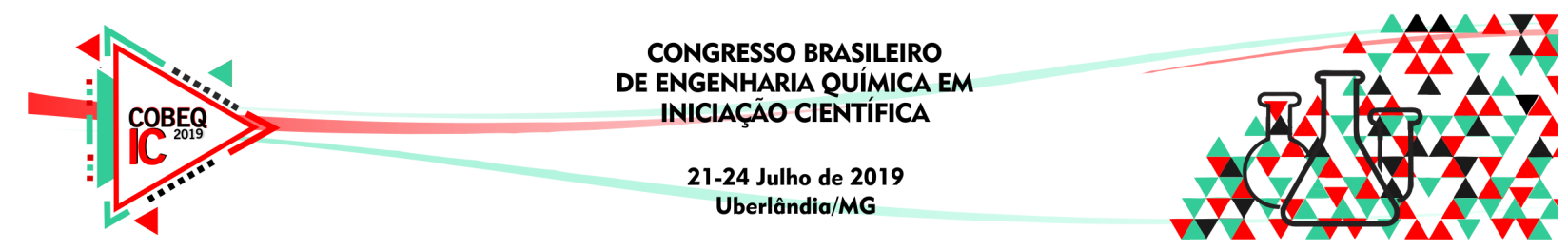

A produção do biodiesel convencional é realizada por uma reação de transesterificação na qual um álcool de cadeia curta (metanol ou etanol) reage com os triacilgliceróis e ácidos graxos livres, convertendo-os em alquil ésteres de ácidos graxos.

Em geral, a reação de transesterificação é catalisada por ácidos $\left(\mathrm{H}_{2} \mathrm{SO}_{4}\right.$ ou $\left.\mathrm{HCl}\right)$, ou bases $(\mathrm{KOH}$ ou $\mathrm{NaOH})$. Apesar de a catálise básica apresentar uma velocidade de reação maior que a catálise ácida, o uso de catalisador ácido muitas vezes é preferível porque ocorre uma reação paralela de saponificação entre álcalis e ácidos graxos livres, presentes em grandes quantidades na biomassa de microalgas, o que reduz o rendimento da transesterificação e emulsiona produto, dificultando sua separação (Fukuda et al., 2001).

\subsection{Transesterificação in situ}

A transesterificação in situ, ou direta, foi proposta por Harrington (1985) e consiste em utilizar o próprio álcool reagente como solvente, dispensando a extração prévia do óleo. Este método se apresenta como uma alternativa interessante para a produção de biodiesel a partir de microalgas, uma vez que possibilita simplificar o processo, aumentar sua produtividade e eliminar uma das etapas de mais alto custo do processo, a extração de lipídios.

A temperatura, a porcentagem de catalisador, o tempo de reação e o volume de álcool que levam às condições ótimas de reação dependem fortemente da espécie de alga e do perfil de lipídios correspondente.

\section{MATERIAIS E MÉTODOS}

\section{1. - Cepa de microalga}

A cepa usada neste trabalho foi a Desmodesmus sp., a qual foi doada pelo Laboratório de Pesquisas com Organismos Aquáticos (LAPOA) do Grupo Integrado de Aquicultura e Estudos Ambientais (GIA) da Universidade Federal do Paraná (UFPR), Curitiba, Brasil. Atualmente, é mantida no laboratório LOPCA/REF-BIORREF da Faculdade de Engenharia Química da UNICAMP e já existem vários estudos que mostram que essa é uma cepa promissora para a produção de biodiesel (Rios et al., 2015). O cultivo foi realizado exclusivamente para a obtenção da biomassa e não foram estudados parâmetros de crescimento. Este cultivo foi realizado em um banco de luzes, agitados por meio de agitação magnética e iluminados $24 \mathrm{~h}$ por dia. A recuperação da biomassa do meio de cultivo foi realizada por meio de centrifugação (marca Eppendorf, modelo 5810R).

\section{2. - Planejamento Experimental}

Com o objetivo de determinar as melhores condições para otimização do rendimento de ésteres na reação de transesterificação in situ, foi realizado um planejamento fatorial fracionário $\left(2^{\mathrm{k}-1}\right)$ com duplicata no ponto central (nível 0) para a estimativa dos contrastes relacionados aos efeitos principais das variáveis independentes. Segundo a literatura, sabe-se que as variáveis independentes de grande importância para o processo são: tipo de álcool 


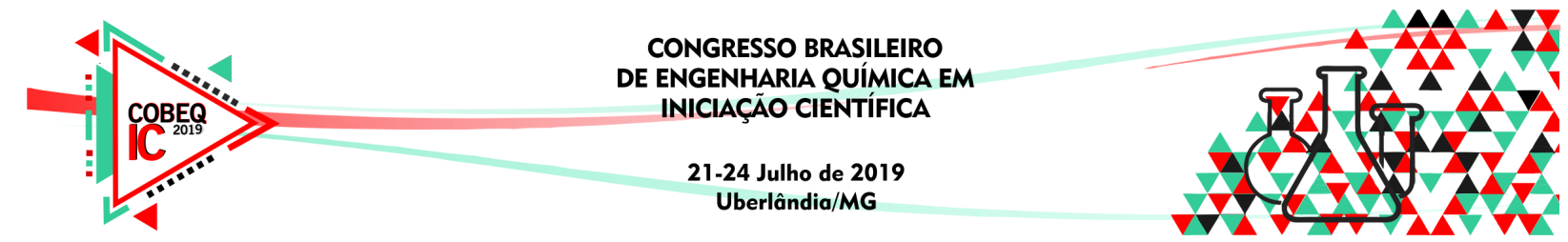

(etanol, metanol), quantidade de catalisador, razão molar óleo:álcool, temperatura e tempo da reação. Com base nesta informação, as variáveis escolhidas para este estudo foram: porcentagem de catalisador, volume de álcool, temperatura e tempo de reação. Na Tabela 1, apresentam-se as faixas das variáveis estudadas.

Tabela 1 - Planejamento experimental em dois níveis.

\begin{tabular}{|c|c|c|c|}
\hline Variável & -1 & 0 & +1 \\
\hline \hline Catalisador $(\%)$ & 20 & 50 & 80 \\
\hline Temperatura $\left({ }^{\circ} \mathrm{C}\right)$ & 70 & 105 & 140 \\
\hline Volume de álcool $(\mathrm{mL})$ & 3 & 5 & 7 \\
\hline Tempo (h) & 0,5 & 3,75 & 7 \\
\hline
\end{tabular}

\section{3. -Reação de Transesterificação in situ}

Em tubos de ensaio de vidro de $50 \mathrm{~mL}$ com tampas rosqueáveis, para cada ponto estudado, foram adicionados cerca de $500 \mathrm{mg}$ de biomassa. Logo após, adicionou-se $1 \mathrm{~mL}$ do solvente (clorofórmio), seguidos do álcool (metanol) e catalisador (ácido sulfúrico) nas quantidades estabelecidas no planejamento experimental. Também foi adicionado um agitador magnético para obter uma mistura homogênea de todos os reagentes durante a reação e, em seguida, colocaram-se os tubos em banho termostático de glicerina, considerando-se a temperatura e o tempo correspondente a cada experimento.

Após os tempos pré-determinados de reação, removeu-se o tubo do banho e o resfriou rapidamente, via banho de gelo, com o objetivo de parar a reação. Depois do resfriamento, realizou-se uma filtração a vácuo com passagem de água $\left(50 \sim 60^{\circ} \mathrm{C}\right)$ e hexano para retirar a biomassa. Repetiu-se a passagem de água e hexano algumas vezes para garantir somente a permanência da biomassa no filtro. A seguir, a fase liquida seguiu um processo de separação de fases em funil de separação, retirando-se a fase mais leve (ésteres) para um tubo previamente pesado. Finalmente, o solvente (hexano) foi, então, evaporado em estufa a $40{ }^{\circ} \mathrm{C}$ e os produtos (ésteres e biomassa) produzidos foram analisados gravimetricamente, até peso constante. Com os produtos pesados, considerou-se o rendimento a partir da massa da amostra e quantidade de lipídeos da microalga, 11,65 $\pm 1,89 \%$, previamente determinada pelo método Bligh \& Dyer (1959).

\section{4. - Análises Cromatográficas}

O perfil de ácidos graxos dos produtos obtidos da reação de transesterificação in situ foi determinado via cromatografia gasosa. Primeiramente, os produtos obtidos foram divididos em 2 tubos (vial $1,5 \mathrm{~mL}$ e tubo $50 \mathrm{~mL}$ ), com a finalidade de realizar a análise do produto bruto e do produto após uma reação de esterificação, respectivamente. Com cerca de $10 \mathrm{mg}$ das amostras em vials, adicionou-se $200 \mu \mathrm{L}$ de uma solução de heptadecanoato (C17) em metanol $0,2 \mathrm{mg} \mathrm{mL}^{-1}$ utilizada como padrão interno, para quantificação, completou-se o 


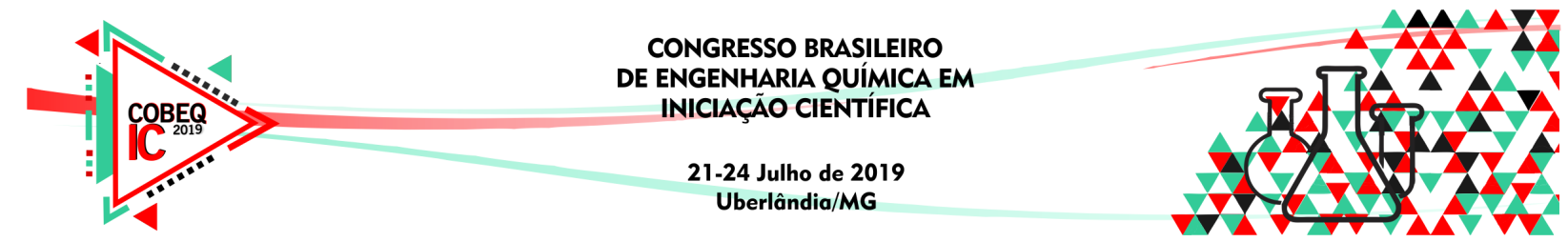

volume com éter de petróleo e armazenou-se as amostras em freezer $\left(-18^{\circ} \mathrm{C}\right)$ até o momento da análise.

Ao restante dos produtos (tubos $50 \mathrm{ml}$ ), adicionou-se $1 \mathrm{~mL}$ da solução padrão e realizou-se uma esterificação baseada na metodologia de Hartman and Lago (1973) para preparação de amostra.

Finalmente, conforme mencionado anteriormente, a composição de ácidos graxos foi determinada por cromatografia gasosa, sendo aqui utilizado detector de ionização de chama (GC / FID 7890A Agilent) com coluna capilar Agilant (50\% cianopropila e 50\% de metilpolissiloxano) e Hidrogênio $\left(\mathrm{H}_{2}\right)$ como gás transportador. A quantificação de ácidos graxos foi baseada na metodologia descrita por Breuer et al. (2013) pela Equação 1.

A porcentagem de cada ácido graxo na amostra foi obtida pela fórmula:

$$
C_{B D}=\frac{\left(A_{t}-A_{I S}\right)}{A_{I S}} x \frac{C_{I S} x V_{I S}}{W}
$$

Onde $A_{t}$ é a área do pico do acido graxo, $A_{I S}$ é a área do padrão interno, $C_{I S}$ é a concentração da solução do padrão interno $\left(\mathrm{mg} \mathrm{mL}^{-1}\right), V_{I S}$ é o volume de padrão $(\mathrm{mL})$ e $W$ é a massa da amostra (mg).

\section{RESULTADOS E DISCUSSÃO}

Na Tabela 2 são apresentados os pontos do planejamento e os respectivos resultados de rendimentos obtidos. Pela análise da Tabela, pode-se observar uma boa distribuição dos resultados e os valores do ponto central estão dentro dos limites de todos os experimentos.

Tabela 2 - Massas e Rendimentos

\begin{tabular}{|c|c|c|c|c|c|c|}
\hline $\begin{array}{c}\text { Ponto do } \\
\text { experimento }\end{array}$ & $\begin{array}{c}\text { Volume de } \\
\text { álcool }(\mathrm{mL})\end{array}$ & $\begin{array}{c}\text { Catalisador } \\
(\mathrm{uL})\end{array}$ & $\begin{array}{c}\text { Temperatura } \\
\left({ }^{\circ} \mathrm{C}\right)\end{array}$ & Tempo (h) & $\begin{array}{c}\text { Rendimento } \\
\left(100 * \text { mg }_{\text {éster }} /\right. \\
\left.\mathrm{mg}_{\text {óleo }}\right)\end{array}$ & $\begin{array}{c}\text { Rendimento } \\
\left(10 *^{*} \text { mgéster/ }\right. \\
\left.\mathrm{g}_{\text {biomassa }}\right)\end{array}$ \\
\hline \hline 1 & 3 & 54 & 70 & 0,50 & 50,05 & 58,31 \\
\hline 2 & 3 & 218 & 70 & 7,00 & 42,73 & 49,79 \\
\hline 3 & 3 & 54 & 140 & 7,00 & 66,63 & 77,62 \\
\hline 4 & 3 & 218 & 140 & 0,50 & 71,08 & 82,80 \\
\hline 5 & 7 & 54 & 70 & 7,00 & 25,16 & 29,32 \\
\hline 6 & 7 & 218 & 70 & 0,50 & 61,53 & 71,69 \\
\hline 7 & 7 & 54 & 140 & 0,50 & 73,63 & 85,78 \\
\hline 8 & 7 & 218 & 140 & 7,00 & 50,49 & 58,82 \\
\hline 9 & 5 & 136 & 105 & 3,75 & 29,39 & 34,24 \\
\hline 10 & 5 & 136 & 105 & 3,75 & 37,35 & 43,51 \\
\hline
\end{tabular}




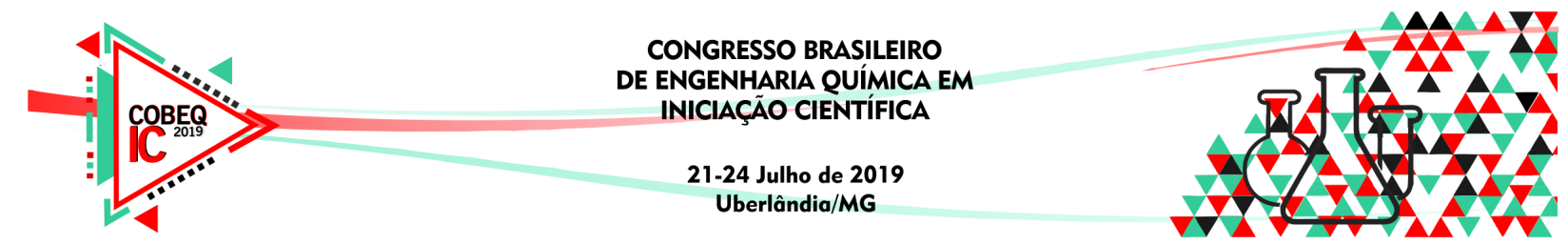

Dentre os rendimentos em ésteres etílicos alcançados na reação, o maior foi de $85,78 \mathrm{mg}$ éster $/ \mathrm{g}_{\text {biomassa }}$ obtido nas condições de: $7 \mathrm{~mL}$ de metanol, $54 \mu \mathrm{L}$ de catalisador $(20 \%)$, $140{ }^{\circ} \mathrm{C}$ e 0,5 h de reação (Ponto 7).

A Tabela 3 mostra o perfil de ácidos graxos das amostras esterificadas para cada ponto do planejamento experimental. Os resultados apresentam as porcentagens de cada ácido em relação ao total de ácidos graxos identificados. As análises cromatográficas realizadas apresentam resultados dentro do esperado, uma vez que as microalgas do gênero Desmodesmus sp. possuem os ácidos palmítico (C16:0) e oleico (C18:1) entre sua composição lipídica majoritária. Não se observou uma mudança significativa nas porcentagens dos ácidos graxos nos pontos do planejamento.

Foram identificados ésteres saturados, monoinsaturados e poli-insaturados, com predomínio dos saturados, majoritariamente o C16:0. O grau de saturação afeta, principalmente, as propriedades de escoamento e estabilidade oxidativa do biocombustível. Pode-se observar pouca diferença entre os diferentes pontos do planejamento, quer dizer que o perfil de ácidos graxos não é significativamente afetado pelas condições da reação.

Tabela 3 - Porcentagem dos ácidos graxos identificados

\begin{tabular}{|l|c|c|c|c|c|c|c|c|c|c|}
\hline \multicolumn{10}{|c|}{ Pontos do experimento } \\
\hline \hline & 1 & 2 & 3 & 4 & 5 & 6 & 7 & 8 & 9 & 10 \\
\hline C15:0 & 0,80 & - & - & - & - & - & - & - & - & - \\
\hline C16:0 & 69,92 & 80,79 & 67,32 & 79,64 & 69,82 & 73,22 & 76,92 & 66,25 & 74,22 & 81,62 \\
\hline C16:1 & 2,72 & - & 3,91 & - & 3,08 & 2,97 & 2,28 & 3,76 & 2,86 & - \\
\hline C16:3 & 0,90 & - & - & - & - & - & - & - & - & - \\
\hline C18:0 & 2,67 & - & 2,88 & - & 2,81 & 2,69 & 3,04 & 3,20 & 2,77 & \\
\hline C18:1 & 16,81 & 19,21 & 20,85 & 16,43 & 18,17 & 16,95 & 13,54 & 20,87 & 16,54 & 18,38 \\
\hline C18:2 & - & - & 2,58 & - & - & - & - & 3,63 & - & - \\
\hline C20:0 & 0,88 & - & - & - & 1,36 & - & - & - & - & - \\
\hline C20:1 & 5,30 & - & 2,45 & 3,93 & 4,76 & 4,17 & 4,21 & 2,28 & 3,60 & - \\
\hline
\end{tabular}

\section{CONCLUSÕES}

A reação de transesterificação in situ foi possível em todas as condições estudadas. Pode-se concluir então que o parâmetro temperatura de reação é o mais significativo na obtenção de ésteres. Uma vez que, os maiores rendimentos foram obtidos nas condições de $140{ }^{\circ} \mathrm{C}$. O tempo da reação teve um efeito significativo negativo, pois o máximo rendimento foi encontrado no nível mínimo do tempo. O maior rendimento foi encontrado nas condições de maior temperatura, maior volume de álcool, menor volume de catalisador e menor tempo,

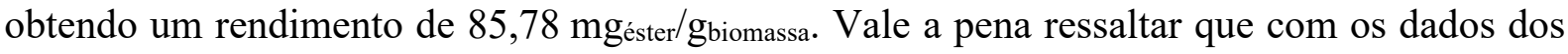
rendimentos obtidos pode ser realizada a análise estatística, a qual será realizada em um trabalho futuro. 


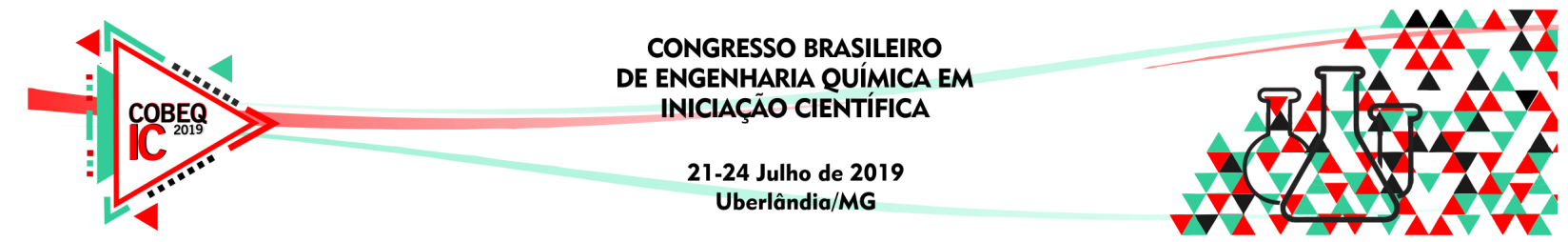

$\mathrm{Na}$ composição de ácidos graxos para os ésteres obtidos, em todas as condições estudadas, houve predominância de componentes saturados, sendo em média $76 \%$ dos ácidos identificados. Essa característica é inerente à espécie e gênero de microalga, pois é consequência direta da composição lipídica, e também é característica relevante que precisa ser considera para a produção e viabilidade de biodiesel.

\section{AGRADECIMENTOS}

Os autores agradecem o financiamento da $\mathrm{CNPq}$ junto com o programa PIBIC/UNICAMP pelo suporte à pesquisa e a Fundação de Amparo à Pesquisa do Estado de São Paulo Processos No 2014/10064-9 e 2015/20630-4.

\section{REFERÊNCIAS}

AOCS Official Method Ce 2-66 Hartmann, L; Lago, LCA. Rapid preparation of fatty cid methyl esters from lipids. Laboratory Practice. July 1973. Pág 475.

BLIGH, E. G.; DYER, W. J. A rapid method of total lipid extraction and purification, Canadian Journal of Biochemistry and Physiology, v. 37, n. 8, 1959.

Braun, J. V.; Santos, V. O. B.; Fontoura, L. A. M.; Pereira, E.; Napp, A.;Seferin, M.;Lima, J.; Ligabue, R.; Vainstein, M. H. GC-FID METHODOLOGY VALIDATION FOR THE FATTY ESTERS CONTENT DETERMINATION IN BIODIESEL WITH HEXADECYL ACETATE AS THE INTENAL STANDARD. Quim. Nova, Vol. 40, No. 9, 1111-1116, 2017. http://dx.doi.org/10.21577/0100-4042.20170103.

Fukuda, H., Kondo, A., Noda, H., 2001. Biodiesel fuel production by transesterification of oils. J. Biosci. Bioeng. 92, 405-416. https://doi.org/10.1016/S1389-1723(01)80288-7

Harrington, Kevin J., D.-E., Catherine, 1985. Transesterification in situ of sunflower seed oil. Ind. Eng. Prod. Res. Dev. 24, 314-318. https://doi.org/10.1021/i300018a027

Oilgae, 2014. Clixoo - Oilgae - Comprehensive Report on Attractive Algae Product Opportunities.

Su, Y., Song, K., Zhang, P., Su, Y., Cheng, J., Chen, X., 2017. Progress of microalgae biofuel's commercialization. Renew. Sustain. Energy Rev. 74, 402-411. https://doi.org/10.1016/j.rser.2016.12.078 